\title{
Cyclin D1 overexpression correlates with poor tumor differentiation and prognosis in gastric cancer
}

\author{
YAN-SHEN SHAN ${ }^{1 *}$, HUI-PING HSU ${ }^{1 *}$, MING-DERG LAI ${ }^{2}$, YU-HSUAN HUNG ${ }^{2}$, \\ CHIH-YANG WANG ${ }^{2}$, MENG-CHI YEN ${ }^{3}$ and YI-LING CHEN ${ }^{4,5}$
}

\begin{abstract}
Departments of ${ }^{1}$ Surgery and ${ }^{2}$ Biochemistry and Molecular Biology, National Cheng Kung University Hospital, College of Medicine, National Cheng Kung University, Tainan 70101; ${ }^{3}$ Department of Emergency Medicine,

Kaohsiung Medical University Hospital, Kaohsiung Medical University, Kaohsiung 80708;

${ }^{4}$ Department of Senior Citizen Service Management and ${ }^{5}$ Senior Citizen Development Center, Chia Nan University of Pharmacy and Science, Tainan 71710, Taiwan, R.O.C.
\end{abstract}

Received November 23, 2015; Accepted April 24, 2017

DOI: $10.3892 / \mathrm{ol} .2017 .6736$

\begin{abstract}
Overexpression of cyclin D is associated with the molecular tumorigenesis of gastric cancer. The purpose of the present study was to investigate the expression of cyclin D in human gastric cancer and to determine the potential correlations between cyclin D expression and clinicopathological characteristics of specific histological types, as well as its prognostic significance. In the present study, the expression of the cyclin D1 (CCND1), cyclin D2 (CCND2) and cyclin D3 $(C C N D 3)$ genes in gastric cancer patients was explored using the Oncomine database, and their correlation with overall survival (OS) and progression-free survival (PFS) was evaluated using Kaplan-Meier analysis. The prognostic significance of CCND1 protein expression was evaluated by western blot analysis of 32 matched specimens of gastric adenocarcinomas and normal tissues obtained from patients treated at the National Cheng Kung University Hospital (Tainan, Taiwan). Analysis of the Oncomine cancer microarray database revealed that $C C N D 1$ gene expression was significantly increased in gastric intestinal-type adenocarcinoma, while $C C N D 2$ was significantly increased in diffuse gastric adenocarcinoma, gastric intestinal-type adenocarcinoma and gastric mixed adenocarcinoma. Kaplan-Meier analysis indicated that overexpression of CCND1 was associated with reduced $\mathrm{OS}$ and $\mathrm{PFS}$. In addition, overexpression of $C C N D 1$ and downregulation of $C C N D 2$ were significantly correlated
\end{abstract}

Correspondence to: Professor Yi-Ling Chen, Department of Senior Citizen Service Management, Chia Nan University of Pharmacy and Science, 60 Erh-Jen Road, Section 1, Jen-Te, Tainan 71710, Taiwan, R.O.C.

E-mail: s5887110@nckualumni.org.tw

*Contributed equally

Key words: gastric cancer, cyclin D1, histological type, overall survival, progression-free survival with receptor tyrosine-protein kinase erb-2-negative tumors and poor differentiation. The ratio of relative $C C N D 1$ expression (expressed as the $C C N D 1 / \beta$-actin ratio) in tumor tissues compared with that in normal tissues was correlated with poor differentiation $(\mathrm{P}=0.0018)$. In summary, $C C N D 1$ overexpression is associated with shorter survival in patients with gastric cancer and with poorly differentiated tumors.

\section{Introduction}

Gastric cancer is the fourth most common type of cancer and the second leading cause of cancer mortality worldwide (1). The most common type of gastric cancer is adenocarcinoma, which is classified into intestinal and diffuse types (2), which develop through distinct pathways (3). Although treating receptor tyrosine-protein kinase erb-2 (Her-2/neu/H2 N/HER2)-overexpressing gastric cancers with trastuzumab has significantly improved patient survival (4), the prognosis of patients with advanced gastric adenocarcinoma is poor; the 5-year survival rate is $<20 \%$ (5), which may in part be due to the lack of prognostic and diagnostic biomarkers. Molecular biomarker expression provides prognostic value and prompts the development of more effective molecular targeted drugs. Genetic and epigenetic alterations of proto-oncogenes and tumor-suppressor genes, including epidermal growth factor receptor and those involved in the phosphatidylinositol 3-kinase/protein kinase $\mathrm{B} / \mathrm{mechanistic}$ target of rapamycin signaling pathway, have been associated with gastric cancer $(6,7)$.

The cyclin D protein family regulates cell cycle progression, which is mediated by their interactions with cyclin-dependent kinases 2, 4 and 6 (8). There have been three isoforms, cyclin D1 (CCND1); cyclin D2 (CCND2); and cyclin D3 (CCND3), identified in humans $(9,10)$. Overexpression of $C C N D 1$ is correlated with tumor differentiation, poor survival and increased metastasis (11-13). Amplification of $C C N D 1$ has been associated with non-small cell lung cancers $(14,15)$, head and neck squamous cell carcinomas (16-18) and pancreatic carcinomas (19). CCND1, CCND2 and CCND3 serve differential roles in tumor cell carcinogenesis that are cell and tissue-type specific (20). 
High levels of $C C N D 2$ expression were observed in ovarian and testicular tumors $(21,22)$, and overexpression of CCND2 has been associated with gastric cancer progression $(23,24)$. In addition, CCND3 has been associated with cell proliferation as well as induction and/or maintenance of terminal differentiation (25). Furthermore, overexpression of $C C N D 1$ and $C C N D 3$ has been identified in malignant melanomas (26), pancreatic cancer (27) and ductal carcinoma of the breast (28). To understand the contribution of CCND1, CCND2 and CCND3 to tumor progression, a detailed analysis of their expression levels in gastric cancer must be explored.

The aim of the present study was to evaluate the expression of CCND1 protein and its correlation with the clinical outcome of patients with gastric cancer. In order to identify the effects of CCND1, CCND2 and CCND3 in cancer cells, their differential roles in gastric cancer were examined. It was hypothesized that increased CCND1 expression may be used as biomarker in patients with gastric cancer. Therefore, data on $C C N D 1, C C N D 2$ and $C C N D 3$ mRNA expression were extracted from the Oncomine database (29) for gastric cancer, and the effect of $C C N D 1, C C N D 2$ and $C C N D 3$ expression level on overall survival (OS) and progression-free survival (PFS) was examined by Kaplan-Meier analysis.

\section{Materials and methods}

Patients. Fresh specimens were collected from 32 patients with gastric adenocarcinoma who underwent radical resection at National Cheng Kung University Hospital (Tainan, Taiwan) between August 2003 and August 2008. The mean age was $60 \pm 11$ years old (range, 35-82 years old; 20 males, 12 females). A total of 32 pairs of cancerous and matched adjacent normal gastric mucosa tissues were collected and analyzed as previously described (30). The Tumor, Node, Metastasis system by the American Joint Committee on Cancer was used for the classification, grading and staging of gastric cancer (31). The specimens were preserved in the Human Biobank within the Research Center of Clinical Medicine of the National Cheng Kung University Hospital (Tainan, Taiwan). All the patients provided written informed consent, and the study was approved by the Institutional Review Board of National Cheng Kung University Hospital (approval no., ER-97-148).

Western blot analysis of CCND1 protein. Total cell lysates were prepared and analyzed by $10 \%$ SDS-PAGE as previously described $(30,32,33)$. Membranes were blocked with $5 \%(\mathrm{w} / \mathrm{v})$ skimmed milk (Merck KGaA, Darmstadt, Germany) for $1 \mathrm{~h}$ at room temperature and incubated with the following primary antibodies overnight at $4^{\circ} \mathrm{C}$ : Anti-CCND1 (cat. no., 2922; dilution, 1:2,000; Cell Signaling Technology, Inc., Danvers, MA, USA) and anti- $\beta$-actin (cat. no., GTX26276; dilution, 1:5,000; GeneTex, Inc., Irvine, CA, USA). Membranes were then incubated for $1 \mathrm{~h}$ at room temperature with peroxidase-conjugated goat anti-rabbit IgG (cat. no. 7074S; 1:3,000; Cell Signaling Technology, Inc.) or peroxidase-conjugated sheep anti-mouse IgG antibody (ECL anti-mouse IgG; cat. no. NA931V; 1:3,000) (Amersham Pharmacia Biosciences, Buckinghamshire, U.K.). Immunodetection was performed using the horseradish peroxidase-based SuperSignal Chemiluminescent Substrate (Pierce; Thermo Fisher Scientific, Inc., Waltham, MA,
USA). For quantification, the bands were measured with the AlphaImager 2200 Imaging System (Alpha Innotech; Bio-Techne, Minneapolis, MN, USA), and the densities of the CCND1 bands were normalized to those of the $\beta$-actin bands. CCND1 expression was quantified and described as a ratio to $\beta$-actin expression (CCND1/ $\beta$-actin ratio).

Bioinformatics and statistical analysis. A search of the Oncomine database (http://www.oncomine.com) (34) was initially conducted to systematically assess the expression level of the $C C N D 1, C C N D 2$ and $C C N D 3$ genes in gastric cancer. For this, normal vs. cancer tissues were compared in the differential analysis. The results were analyzed for their P-values, fold change and cancer subtype. The prognostic value of the $C C N D 1, C C N D 2$ and $C C N D 3$ genes in gastric cancer was also analyzed using the Kaplan-Meier Plotter (http://kmplot.com/analysis/), as described previously (35). The following settings were used for the analysis: 'Overall survival'; 'progression-free survival'; 'autoselect best cutoff'; 'censore at threshold all' (patients surviving over the selected threshold are censored instead of excluded); 'tumor stage all'; 'tumor stage $\mathrm{T}$ all'; 'tumor stage $\mathrm{N}$ all'; 'tumor stage $\mathrm{M}$ all'; 'grade all'; 'Lauren classification all' (2); 'differentiation all'; and 'moderate and poor differentiation'. Tumors were classified according to WHO histopathological type (36). Three cyclin D genes probe sets were available: 208712 at at $C C N D 1$, 200953_s_at at CCND2 and 201700_at at CCND3, and patients were split according to median expression or to expression at best cut-off for each probe. A total of 1,065 patients with gastric cancer were assessed using a Kaplan-Meier plot (36) and HER2 status was identified using the gene chip probe set 216836_s_at, as previously described (37). The hazard ratio (HR) 95\% confidence intervals and logrank P-values were calculated and described. $\mathrm{P}<0.05$ was considered to indicate a statistically significant difference. The data were extracted from the Oncomine database and Kaplan-Meier Plotter between March 2015 and August 2015. Finally, the association between CCND1 protein expression, assessed according to previously published protocols $(30,38)$ (CCND1/ $\beta$-actin ratio), and differentiation type (moderate and poor differentiation) in fresh specimens derived from patients with gastric adenocarcinoma was assessed using the Student's t-test. The statistical differences between two groups were assessed, and $\mathrm{P}<0.05$ was considered to indicate a statistically significant difference. Statistical analysis was performed using GraphPad Prism 5 (GraphPad Software, Inc., La Jolla, CA, USA).

\section{Results}

Analysis of CCND1, CCND2 and CCND3 gene expression. Data for $C C N D 1, C C N D 2$ and $C C N D 3$ transcript expression were extracted from the Oncomine database for gastric cancer, focusing on cancer vs. normal patient datasets. The statistical significance, fold change, patient number, and type of tissues that displayed upregulation or downregulation of CCND1, $C C N D 2$ and $C C N D 3$ gene expression were also analyzed in normal vs. cancer tissues from the Oncomine database. The Derrico and Cho datasets obtained from Oncomine is embedded in the NCBI GEO database (https://www.ncbi.nlm. nih.gov/geo/) at accession numbers GSE13911 and GSE13861, 

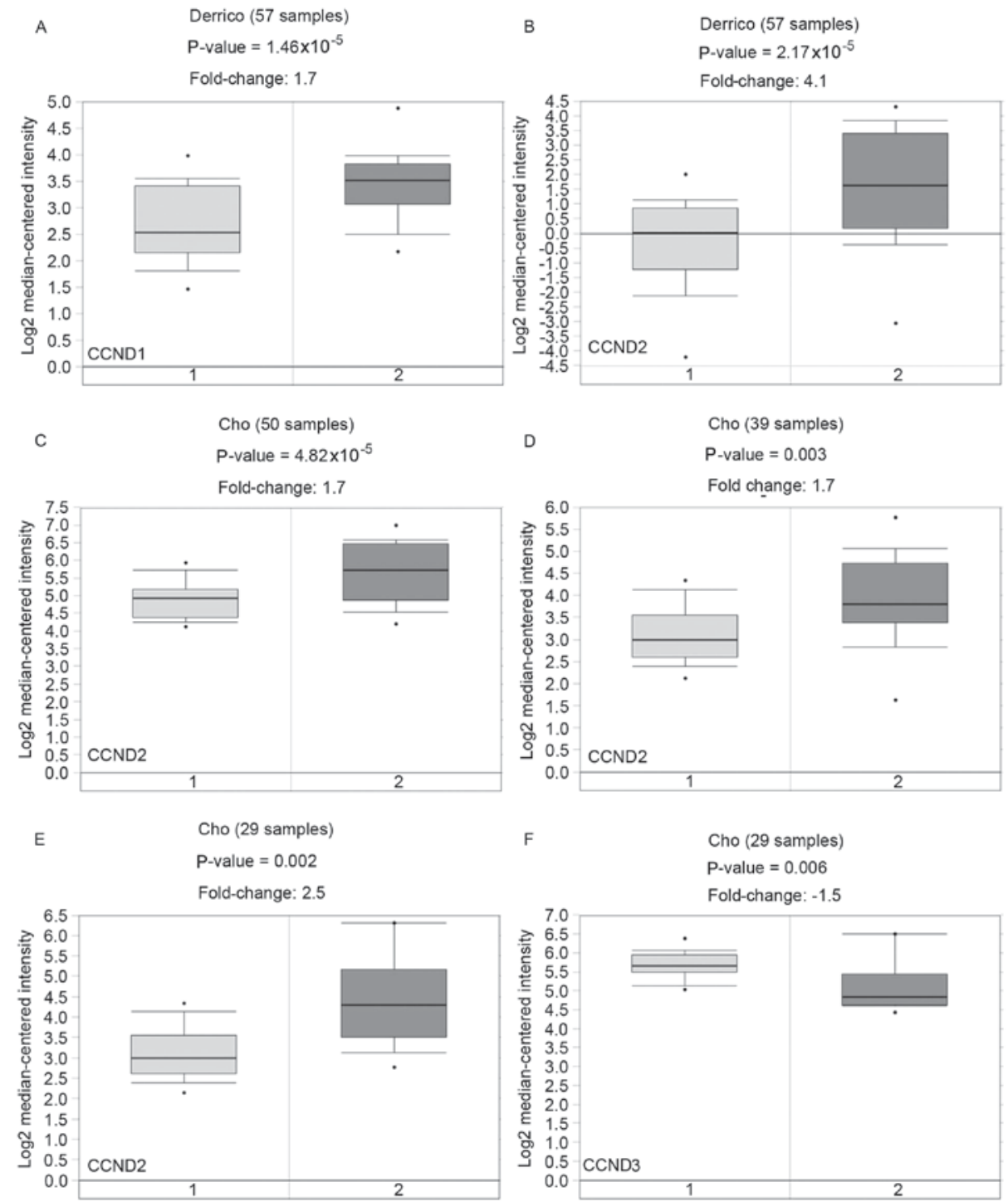

Figure 1. Gene expression of $C C N D 1, C C N D 2$ and $C C N D 3$ from Oncomine database in gastric cancer. $C C N D 1$ was overexpressed in the Derrico dataset. $C C N D 2$ was overexpressed in the Derrico and Cho datasets. CCND3 was underexpressed in the Cho dataset. The expression patterns of (A) CCND1, (B-E) CCND2 and (F) CCND3 in gastric cancer datasets were obtained from the Oncomine database. (A and B) 1, gastric mucosa; 2, gastric intestinal type adenocarcinoma. (C) 1, gastric tissue; 2, diffuse gastric adenocarcinoma. (D) 1, gastric tissue; 2, gastric intestinal type adenocarcinoma. (E and F) 1, gastric tissue; 2, gastric mixed adenocarcinoma. CCND, cyclin D.

respectively (Fig. 1). Overexpression and downregulation of the $C C N D 1, C C N D 2$ and $C C N D 3$ genes were identified in gastric cancer (Fig. 1). To determine the clinical relevance of $C C N D 1$, $C C N D 2$ and $C C N D 3$ expression in human gastric cancer, their expression profiles in the oncomine cancer microarray database were analyzed. Information on the expression of CCND1, $C C N D 2$ and $C C N D 3$ in normal and cancerous gastric tissues was compiled from all of the microarray studies in the database $(39,40)$. The histological type of gastric adenocarcinoma was divided into intestinal, diffuse and mixed types (2). As demonstrated in Fig. 1A, CCND1 expression was significantly increased in gastric intestinal-type adenocarcinoma of gastric cancer (40). CCND2 expression was significantly increased in several types of gastric cancer, including diffuse gastric adenocarcinoma, gastric intestinal-type adenocarcinoma and gastric mixed adenocarcinoma (Fig. 1B-E) $(39,40)$. By contrast, CCND3 expression was significantly decreased in gastric mixed adenocarcinoma (Fig. 1F) (39). Oncomine analysis of neoplastic vs. normal tissue revealed that $C C N D 1$ and $C C N D 2$ were overexpressed in gastric cancer from the GSE13911 and GSE13861 datasets, respectively.

Association of CCND1, CCND2 and CCND3 expression with $O S$ and PFS in patients with gastric cancer. To analyze the association of $C C N D 1, C C N D 2$ and $C C N D 3$ expression with gastric cancer patient survival, Kaplan-Meier survival curves were constructed (Fig. 2). A significant association was identified between $C C N D 1, C C N D 2$ and $C C N D 3$ mRNA and survival $(\mathrm{P}<0.05$, log-rank test). Overexpression of CCND1 (Fig. 2A and B) was correlated with lower OS and PFS. By contrast, $C C N D 2$ overexpression was correlated with increased survival (Fig. 2C and D). Overexpression of $C C N D 3$ was correlated with lower OS and PFS (Fig. 2E and F).

Effect of CCND1, CCND2 and CCND3 expression on gastric cancer patient survival by differentiation types. When the 

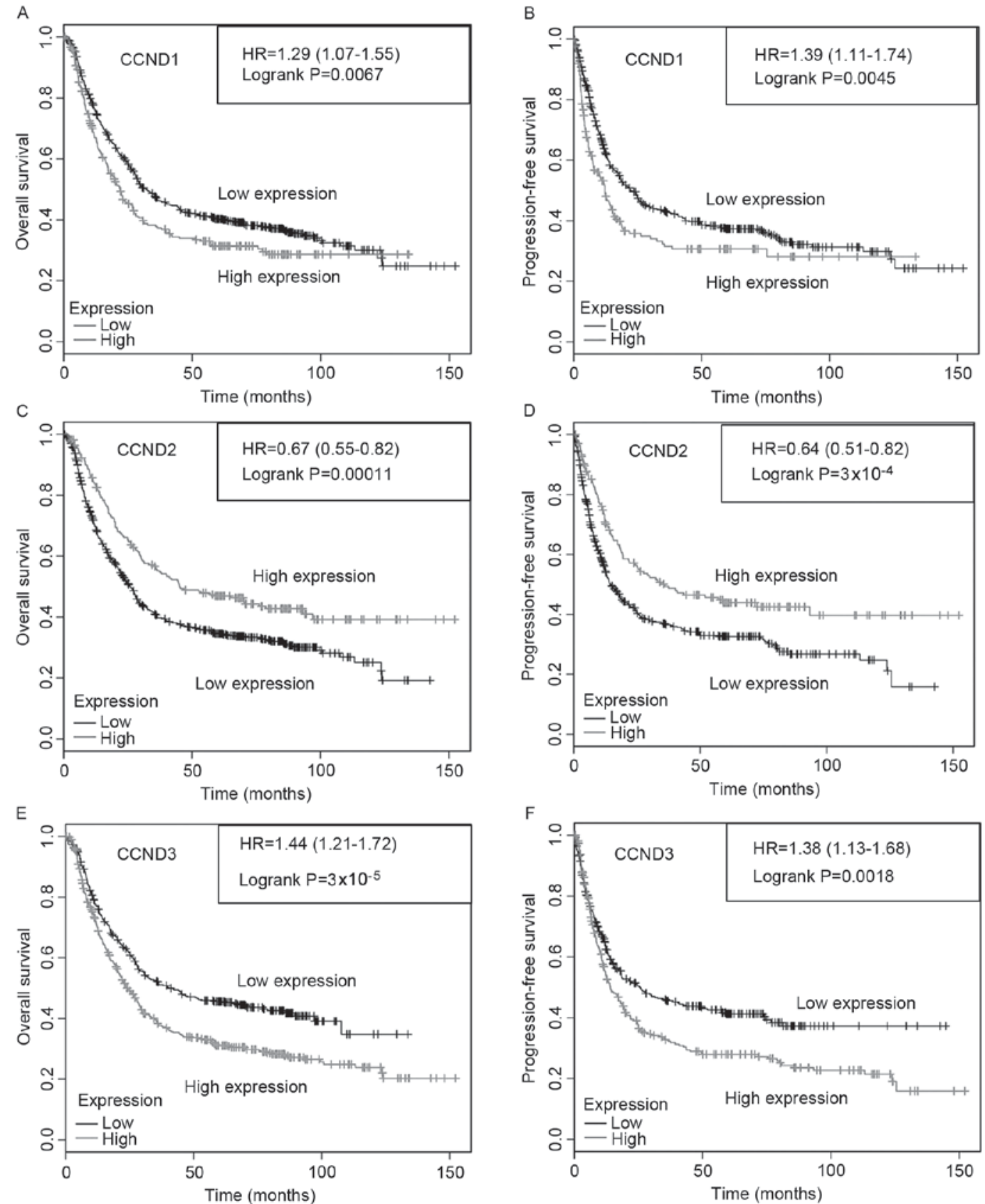

Figure 2. Kaplan-Meier survival curves regarding $C C N D 1, C C N D 2$ and $C C N D 3$ gene expression in patients with gastric cancer. High expression of $C C N D 1$ was associated with lower OS (A) and PFS (B). Low expression of CCND2 was correlated with lower OS (C) and PFS (D). High expression of CCND3 was correlated with lower OS (E) and PFS (F). The total number of patients in the low- and high-expression groups, as well as the HR and P-values (log-rank), are included. CCND, cyclin D; HR, hazard ratio; OS, overall survival; PFS, progression-free survival.

analysis was restricted by differentiation type, significant differences in OS and PFS were observed for the expression of the CCND1, CCND2 and CCND3 genes in patients with moderately and poorly differentiated tumors. Specifically, high CCND1 expression was not associated with PFS compared with that of patients with low CCND1 expression in patients with moderately differentiated gastric cancer (Fig. 3A), but in low CCND1 expression was significantly $(\mathrm{P}=0.0079)$ longer associated with PFS compared with that of patients with high CCND1 expression in patients with poorly differentiated tumors (Fig. 3B). By contrast, high CCND2 expression was associated with a significantly poorer $(\mathrm{P}=0.032) \mathrm{PFS}$ in patients with moderately differentiated tumors (Fig. 3C), but not in those with poorly differentiated gastric cancer (Fig. 3D). Additionally, low CCND3 expression was associated with significantly $(\mathrm{P}=0.039)$ longer $\mathrm{PFS}$ in patients with moderately differentiated tumors (Fig. 3E), but not in patients with poorly differentiated gastric cancer (Fig. 3F). The results demonstrated that the high expression of CCND1 was significantly correlated with poor differentiation and poor survival, while high expression of CCND2 and CCND3 was significantly correlated with moderate differentiation and poor survival.

Association of CCND1, CCND2 and CCND3 expression with gastric cancer patient survival by HER2 status. HER2 

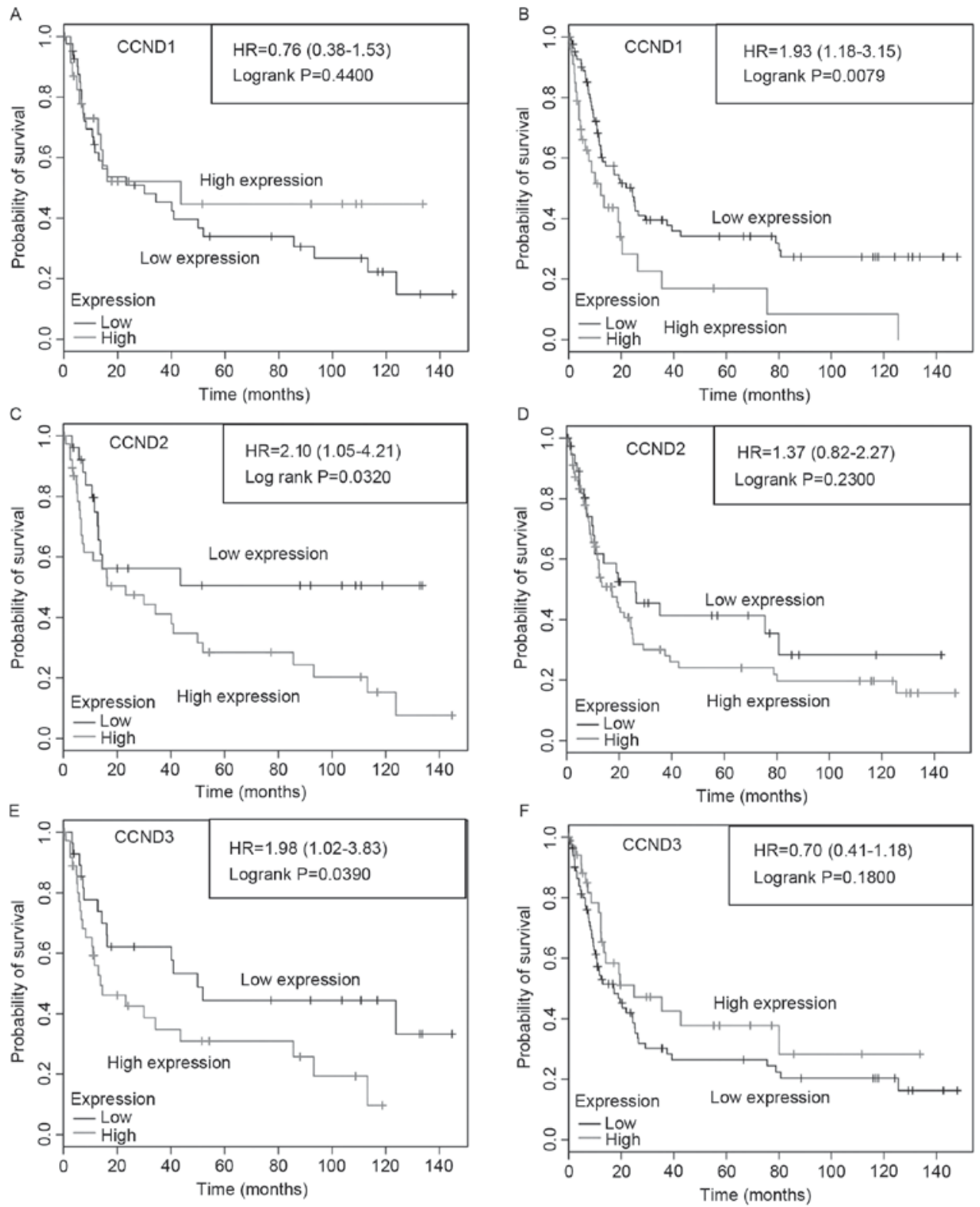

Figure 3. Progression-free survival of patients with moderately and poorly differentiated gastric cancers by $C C N D 1, C C N D 2$ and $C C N D 3$ gene expression CCND1 expression was associated with PFS in patients with moderately (A) and poorly (B) differentiated gastric cancer. CCND2 expression was associated with PFS in patients with moderately (C) and poorly (D) differentiated gastric cancer. CCND3 expression was associated with PFS in patients with moderately (E) and poorly (F) differentiated gastric cancer. The total number of patients in the low- and high-expression groups, as well as the HR and P-values (log-rank), are included. CCND, cyclin D; HR, hazard ratio; OS, overall survival; PFS, progression-free survival.

overexpression has been correlated with poor outcomes and a more aggressive disease (41); however, the association between HER2 status and the prognosis of patients with gastric cancer remains controversial (42). To analyze the association of CCND1, CCND2 and CCND3 expression and HER2 status with survival, Kaplan-Meier PFS curves of PFS stratified by CCND1, CCND2 and CCND3 mRNA expression in HER2-negative and HER2-positive tumors were constructed. Overexpression of CCND1 (Fig. 4A and B) was associated with reduced PFS in HER2-negative and HER2-positive tumors. By contrast, reduced CCND2 expression was associated with lower PFS in patients with either HER2-negative or HER2-positive tumors (Fig. 4C and D). Overexpression of $C C N D 3$ (Fig. 4E and F) was associated with reduced PFS in HER2-negative and HER2-positive tumors. These results indicated that $C C N D 1, C C N D 2$ and $C C N D 3$ expression, and HER2 status were associated with survival.

Effects of CCND1, CCND2 and CCND3 expression on the PFS of patients with poorly differentiated tumors by HER2 status. When the analysis was limited to those patients with gastric cancer with poorly differentiated tumors and was restricted by HER 2 status, significant differences in PFS were observed between high and low expression levels of CCND1, CCND2 and $C C N D 3$ (Fig. 5). Low CCND1 expression was associated with significantly longer PFS in patients with poorly differentiated, HER2-negative tumors (Fig. 5A), but not in those with poorly differentiated, HER2-positive tumors (Fig. 5B). By contrast, low CCND2 expression was associated with significantly poorer PFS in patients with poorly differentiated, 

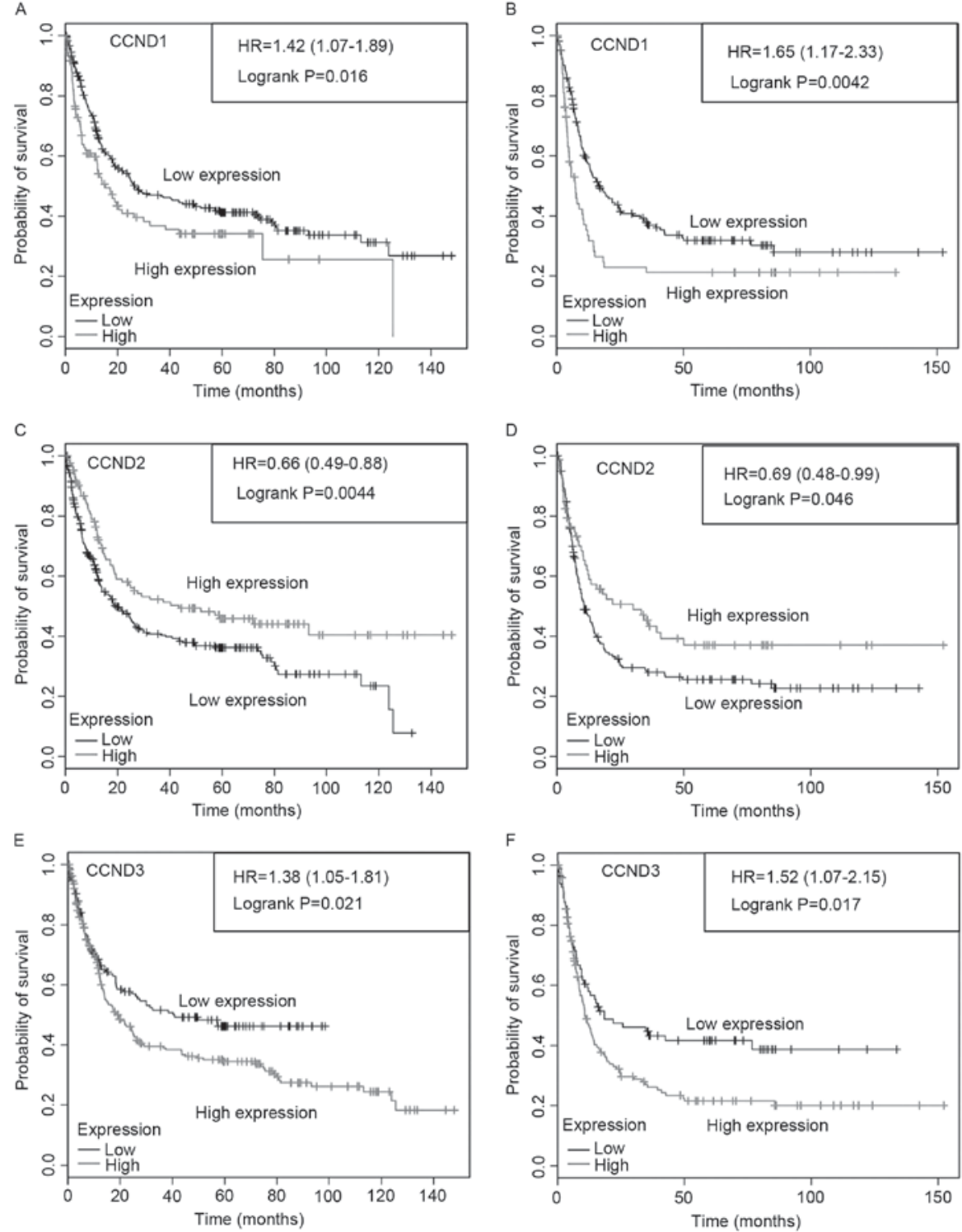

Figure 4. Progression-free survival of patients with gastric cancer with by HER2 status, and CCND1, CCND2 and CCND3 gene expression. Progression-free survival of patients with (A, C and E) HER2-negative or (B, D and F) HER2-positive tumors by (A and B) CCND1, (C and D) CCND2 and (E and F) CCND3 expression. The total number of patients in the low- and high-expression groups, as well as the HR and P-values (logrank), are included. CCND, $c y c l i n D ; \mathrm{HR}$, hazard ratio; HER2, receptor tyrosine-protein kinase erb-2.

HER2-negative tumors (Fig. 5C), but not in those with poorly differentiated, HER2-positive tumors (Fig. 5D). PFS was not affected by CCND3 gene expression level in poorly differentiated, HER2-negative or HER2-positive tumors (Fig. 5E and F, respectively). However, moderately differentiated tumors were not analyzed in the present study due to the small number of patients. Overexpression of CCND1 was significantly correlated with HER2-negative tumors, poor differentiation and poor survival. Additionally, downregulation of CCND2 was significantly correlated with poor differentiation, HER2-negative tumor status and poor survival. Taken together, these results suggest that overexpression of CCND1 is predictive of a poor prognosis and serves an important role in poorly differentiated, HER2-negative gastric tumors.

CCND1 protein expression in clinical samples of gastric cancer tissues. CCND1 expression was also compared in moderately and poorly differentiated gastric cancer, and CCND1 protein expression was examined by western blot analysis in tumor and adjacent normal gastric tissues of 32 patients. All 32 cases of gastric cancer were adenocarcinomas, including 13 moderately differentiated and 19 poorly differentiated tumors (Table I). Analysis of the relative expression of CCND1 in moderately (Fig. 6A) and poorly (Fig. 6B) differentiated tissues indicated that the CCND1/ $\beta$-actin ratio in poorly differentiated samples was significantly greater compared with that in moderately differentiated samples $(\mathrm{P}<0.0018)$ (Fig. 6C). In summary, the overexpression of CCND1 is associated with poorly differentiated gastric cancer.

\section{Discussion}

Analysis of $C C N D 1, C C N D 2$ and $C C N D 3$ expression in human gastric cancer identified that overexpression of 

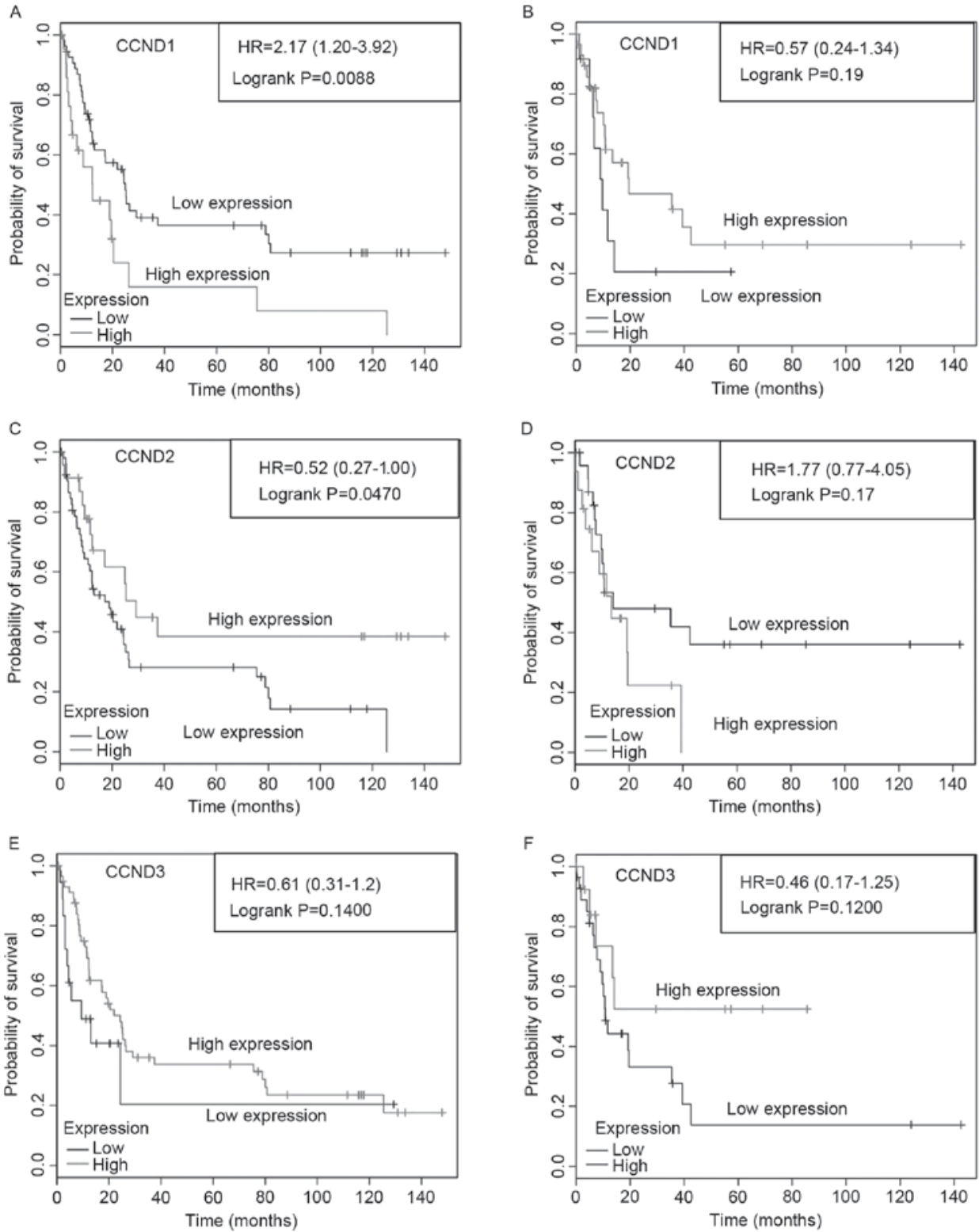

Figure 5. Progression-free survival of patients with poorly differentiated gastric cancer by HER2 expression, and CCND1, CCND2 and CCND3 gene expression. Progression-free survival of patients with (A, C and E) poorly differentiated, HER2-negative or (B, D and F) poorly differentiated, HER2-positive gastric cancer by (A and B) $C C N D 1$, (C and D) $C C N D 2$ and (E and F) $C C N D 3$ expression. The total number of patients in the low- and high-expression groups, as well as the HR and P-values (logrank), are included. CCND, cyclin D; HR, hazard ratio; HER2, receptor tyrosine-protein kinase erb-2.

CCND1 was associated with poor survival of patients with poorly differentiated gastric cancer. In addition, the effect of CCND1 overexpression in patients with HER2-negative tumors was correlated with poor outcomes. The present study suggests that the overexpression of $C C N D 1$, but not of $C C N D 2$ or $C C N D 3$, in poorly differentiated gastric cancer is closely associated with lower survival rates. CCND2 and CCND3 expression were associated with moderate differentiation. Consistent with these results, the present study identified that the CCND1 protein is overexpressed in poorly differentiated gastric tumors. Thus, CCND1 serves a prognostic role in tumor progression, and is involved in the regulation of tumor cell differentiation.

Oncomine analysis identified a strong correlation between $C C N D 1$ and $C C N D 2$ gene expression and certain subtypes of gastric cancer in the present study. Histologically, gastric cancer is divided into two types in the Lauren classification: Intestinal and diffuse (2). In the present study, gastric intestinal-type adenocarcinomas were associated with CCND1 expression, while diffuse gastric adenocarcinoma, gastric intestinal-type adenocarcinoma and gastric mixed adenocarcinoma types were associated with $C C N D 2$ expression. Notably, a previous study suggested that promoter hypermethylation of $C C N D 2$ caused the loss of CCND2 function in gastric cell lines and primary gastric carcinomas (43). In addition, CCND2 protein expression was not detected in KATOIII, AGS, MKN45 or N87 cell lines (43). It has been suggested that hypermethylation of the CCND2 promoter occurs in breast (44), prostate (45) and gastric cancer (43). To date, there have been few studies investigating $C C N D 2$ promoter hypomethylation in colon cancer (46). These studies explain that CCND2 overexpression was 
Table I. Demographics and histopathological data of patients with gastric cancer.

\begin{tabular}{lc}
\hline Characteristic & No. of patients (\%) \\
\hline Patients with gastric cancer & $32(100)$ \\
Mean age \pm standard deviation, years & $60 \pm 11$ \\
Sex & \\
Male & $20(62)$ \\
Female & $12(38)$ \\
Histological differentiation & \\
Moderate & $13(40)$ \\
Poor & $19(60)$ \\
Lauren's classification & \\
Intestinal & $15(47)$ \\
Diffuse & $12(37)$ \\
Mixed & $5(16)$ \\
American Joint Committee on & \\
cancer tumor node metastasis stage & \\
I & $7(22)$ \\
II & $9(28)$ \\
III & $12(38)$ \\
IV & $4(12)$ \\
\hline
\end{tabular}

an early event noted in colon polyps (47), and possibly the overexpression of CCND2, but not of CCND1 or CCND3, was associated with metastatic tumors (46). As patients with diffuse gastric cancer exhibit poorer prognoses and higher incidences of metastasis compared with those of patients with intestinal type tumors $(48,49)$, it is possible that CCND2 overexpression in diffuse gastric adenocarcinoma is also associated with promoter hypomethylation in the early stage, and reveals a potential metastatic role for CCND2.

The Kaplan-Meier analysis of the present study identified correlations between $C C N D 1, C C N D 2$ and $C C N D 3$ gene expression and clinical outcomes. An elevated expression of CCND1 was significantly associated with poor differentiation and poorer PFS. Clinically, the major histological types of metastatic gastric cancer include poorly differentiated and signet ring adenocarcinomas $(50,51)$. CCNDl overexpression is associated with shorter patient survival in mantle cell lymphoma and head and neck squamous cell carcinoma $(11,12)$. In addition, $C C N D 1$ overexpression is often associated with increased metastasis, which is consistent with the ability of CCND1 to enhance migration and invasion $(12,52)$. The expression and potential roles of $C C N D 1$ in gastric cancer have been investigated, and previous studies have demonstrated the manner in which it contributes to carcinogenesis (53-58). For example, direct evidence that CCND1 serves an essential role in the cell proliferation of gastric cancer cell lines has been presented (54). Evidence has also demonstrated that increased CCND1 expression was associated with decreased OS in patients with resected gastric adenocarcinoma (59).

The majority of tumor markers are produced at much higher levels in cancer cells than in normal cells, and may be identified in the blood, urine or tumor tissues of patients

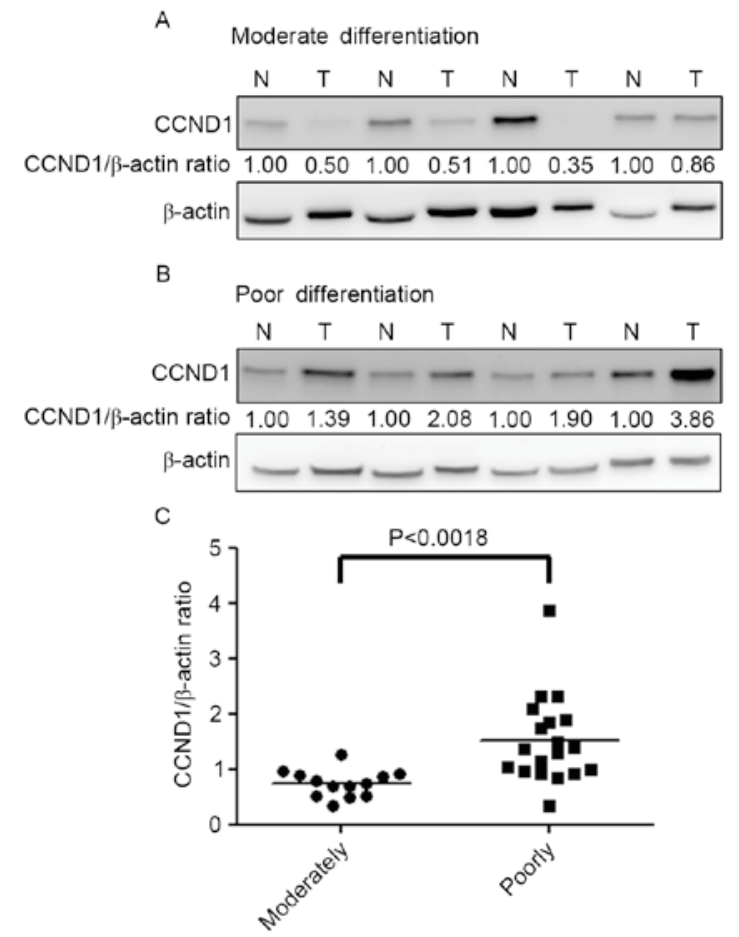

Figure 6. Correlation between differentiation type and CCND1 protein expression in gastric cancer. The tumor/normal ratio of CCND1 expression (the ratio of CCND1 expression in specimens from gastric cancer relative to that in corresponding normal gastric tissues) was determined by western blot analysis. CCND1 expression is presented relative to that of $\beta$-actin (CCND1/ $\beta$-actin ratio). (A) CCND1 expression was measured in moderately differentiated tumors from gastric cancer and normal stomach tissues. (B) CCND1 expression was measured in specimens of poorly differentiated gastric cancer and normal stomach tissues. (C) The CCND1/ $\beta$-actin ratio of 32 samples was compared with that from moderately and poorly differentiated tumors $(\mathrm{P}=0.0018)$. $\mathrm{N}$, normal tissue; $\mathrm{T}$, gastric cancer tissue; CCND1, cyclin D1

with cancer (60-62). Thus, tumor marker analysis may reflect the various stages of the cancer and may assist clinicians in the planning and monitoring of cancer treatment. The present study attempted to identify CCND1 as a prognostic biomarker of poorly differentiated gastric cancer. In a previous study, inhibition of CCND1 using specific targeting was presented as a novel gastric cancer therapy (56). Amplification of CCND1 causes resistance to certain cytotoxic drugs and targeted therapies, including gefitinib and tamoxifen $(63,64)$, and is a potential predictor of resistance to cancer therapy in breast cancer (65). In addition, HER2 is overexpressed and/or gene-amplified in gastric cancer, although numerous studies have yielded inconsistent data regarding the prognostic relevance of HER2 (66). Whereas certain studies demonstrated that HER2 positivity was associated with significantly poor prognosis (66), other studies identified no association between HER2 status and prognosis (66). In a previous study, 7-17\% of patients with gastric cancer were HER2-positive and, thus, suitable candidates for trastuzumab therapy (67). In addition, amplification of CCND1 was revealed in $17.4 \%$ of gastric cancers (60). Clinically, intra-tumor heterogeneity often results in failure of gene therapy and targeted therapy in gastric adenocarcinoma (60). In the present study, the heterogeneity of the potential target genes, $C C N D 1, C C N D 2$ and $C C N D 3$, was systematically analyzed. To identify the most prevalent 
molecular targets in poorly differentiated gastric cancer, the patient outcomes relative to CCND1 overexpression and HER2 status were determined. The results imply that CCND1 overexpression of poorly differentiated gastric cancer causes resistance to certain cytotoxic drugs and targeted therapies.

In conclusion, to the best of our knowledge, the present study is the first to suggest that overexpression of CCND1 protein is correlated with lower PFS in poorly differentiated gastric cancer. These results demonstrate that CCND1, but not CCND2 or CCND3, is overexpressed in human gastric carcinoma, and that the expression of this protein is correlated with tumor differentiation. Thus, CCND1 expression is a valuable prognostic indicator for gastric cancer.

\section{Acknowledgements}

The present study was supported by grants from the Ministry of Science and Technology of Taiwan (grant nos. MOST 102-2311-B041-001 and MOST 103-2311-B-041-001). The authors would like to thank the Human Biobank, the Research Center of Clinical Medicine and the National Cheng Kung University Hospital for their support.

\section{References}

1. Jemal A, Bray F, Center MM, Ferlay J, Ward E and Forman D: Global cancer statistics. CA Cancer J Clin 61: 69-90, 2011.

2. Lauren P: The two histological main types of gastric carcinoma: Diffuse and so-called intestinal-type carcinoma. An attempt at a histo-clinical classification. Acta Pathol Microbiol Scand 64: 31-49, 1965.

3. Correa P: Human gastric carcinogenesis: A multistep and multifactorial process-first American cancer society award lecture on cancer epidemiology and prevention. Cancer Res 52: 6735-6740, 1992.

4. Bang YJ, Van Cutsem E, Feyereislova A, Chung HC, Shen L, Sawaki A, Lordick F, Ohtsu A, Omuro Y, Satoh T, et al: Trastuzumab in combination with chemotherapy versus chemotherapy alone for treatment of HER2-positive advanced gastric or gastro-oesophageal junction cancer (ToGA): A phase 3 , open-label, randomised controlled trial. Lancet 376: 687-697, 2010.

5. Nagini S: Carcinoma of the stomach: A review of epidemiology, pathogenesis, molecular genetics and chemoprevention. World J Gastrointest Oncol 4: 156-169, 2012.

6. Yang W, Raufi A and Klempner SJ: Targeted therapy for gastric cancer: Molecular pathways and ongoing investigations. Biochim Biophys Acta 1846: 232-237, 2014.

7. Matsuoka T and Yashiro M: The role of PI3K/Akt/mTOR signaling in gastric carcinoma. Cancers 6: 1441-1463, 2014.

8. Matsushime H, Ewen ME, Strom DK, Kato JY, Hanks SK, Roussel MF and Sherr CJ: Identification and properties of an atypical catalytic subunit (p34PSK-J3/cdk4) for mammalian D type G1 cyclins. Cell 71: 323-334, 1992.

9. Büschges R, Weber RG, Actor B, Lichter P, Collins VP and Reifenberger G: Amplification and expression of cyclin D genes (CCND1, CCND2 and CCND3) in human malignant gliomas. Brain Pathol 9: 435-433, 1999.

10. Musgrove EA, Caldon CE, Barraclough J, Stone A and Sutherland RL: Cyclin D as a therapeutic target in cancer. Nat Rev Cancer 7: 558-572, 2011.

11. Jares P, Colomer D and Campo E: Genetic and molecular pathogenesis of mantle cell lymphoma: Perspectives for new targeted therapeutics. Nat Rev Cancer 7: 750-762, 2007.

12. Thomas GR, Nadiminti H and Regalado J: Molecular predictors of clinical outcome in patients with head and neck squamous cell carcinoma. Int J Exp Pathol 86: 347-363, 2005.

13. van Diest PJ, Michalides RJ, Jannink L, van der Valk P, Peterse HL, de Jong JS, Meijer CJ and Baak JP: Cyclin D1 expression in invasive breast cancer. Correlations and prognostic value. Am J Pathol 150: 705-711, 1997.
14. Jin $\mathrm{ML}$, Inoue $\mathrm{S}$, Umemura $\mathrm{T}$, Moriya J, Arakawa $\mathrm{M}$, Nagashima K and Kato H: Cyclin D1, p16 and retinoblastoma gene product expression as a predictor for prognosis in non-small cell lung cancer at stages I and II. Lung Cancer 34: 207-218, 2001

15. Yamanouchi H, Furihata M, Fujita J, Murakami $H$, Yoshinouchi T, Takahara J and Ohtsuki Y: Expression of cyclin $E$ and cyclin D1 in non-small cell lung cancers. Lung Cancer 31: 3-8, 2001

16. Ikeguchi M, Sakatani T, Ueta T and Kaibara N: Cyclin D1 expression and retinoblastoma gene protein (pRB) expression in esophageal squamous cell carcinoma. J Cancer Res Clin Oncol 127: 531-536, 2001.

17. Izzo JG, Papadimitrakopoulou VA, Li XQ, Ibarguen H, Lee JS, Ro JY,El-Naggar A, Hong WK and Hittelman WN: Dysregulated cyclin D1 expression early in head and neck tumorigenesis: In vivo evidence for an association with subsequent gene amplification. Oncogene 17: 2313-2322, 1998

18. Bartkova J, Lukas J, Müller H, Strauss M, Gusterson B and Bartek J: Abnormal patterns of D-type cyclin expression and G1 regulation in human head and neck cancer. Cancer Res 55: 949-956, 1995.

19. Gansauge S, Gansauge F, Ramadani M, Stobbe H, Rau B, Harada N and Beger HG: Overexpression of cyclin D1 in human pancreatic carcinoma is associated with poor prognosis. Cancer Res 57: 1634-1637, 1997.

20. Carthon BC, Neumann CA, Das M, Pawlyk B, Li T, Geng Y and Sicinski P: Genetic replacement of cyclin D1 function in mouse development by cyclin D2. Mol Cell Biol 25: 1081-1088, 2005.

21. Choi D, Yoon S, Lee E, Hwang S, Yoon B and Lee J: The expression of pseudogene cyclin D2 mRNA in the human ovary may be a novel marker for decreased ovarian function associated with the aging process. J Assist Reprod Genet 18: 110-113, 2001

22. Bartkova J, Rajpert-De Meyts E, Skakkebaek NE and Bartek J: D-type cyclins in adult human testis and testicular cancer: Relation to cell type, proliferation, differentiation, and malignancy. J Pathol 187: 573-581, 1999.

23. Takano Y, Kato Y, Masuda M, Ohshima Y and Okayasu I: Cyclin D2, but not cyclin D1, overexpression closely correlates with gastric cancer progression and prognosis. J Pathol 189: 194-200, 1999.

24. Yu J, Leung WK, Ng EK, To KF, Ebert MP, Go MY, Chan WY, Chan FK, Chung SC, Malfertheiner P, et al: Effect of helicobacter pylori eradication on expression of cyclin D2 and p27 in gastric intestinal metaplasia. Aliment Pharmacol Ther 15: 1505-1511, 2001.

25. Bartkova J, Lukas J, Strauss M and Bartek J: Cyclin D3: Requirement for G1/S transition and high abundance in quiescent tissues suggest a dual role in proliferation and differentiation. Oncogene 17: 1027-1037, 1998.

26. Flørenes VA, Faye RS, Maelandsmo GM, Nesland JM and Holm R: Levels of cyclin D1 and D3 in malignant melanoma: Deregulated cyclin D3 expression is associated with poor clinical outcome in superficial melanoma. Clin Cancer Res 6: 3614-3620, 2000.

27. Ito $\mathrm{Y}$, Takeda T, Wakasa $\mathrm{K}$, Tsujimoto $\mathrm{M}$ and Matsuura N: Expression and possible role of cyclin D3 in human pancreatic adenocarcinoma. Anticancer Res 21: 1043-1048, 2001.

28. Wong SC, Chan JK, Lee KC and Hsiao WL: Differential expression of p16/p21/p27 and cyclin D1/D3 and their relationships to cell proliferation, apoptosis and tumour progression in invasive ductal carcinoma of the breast. J Pathol 194: 35-42, 2001.

29. Rhodes DR, Kalyana-Sundaram S, Mahavisno V, Varambally R, Yu J, Briggs BB, Barrette TR, Anstet MJ, Kincead-Beal C, Kulkarni P, et al: Oncomine 3.0: Genes, pathways, and networks in a collection of 18,000 cancer gene expression profiles. Neoplasia 9: 166-180, 2007.

30. Shan YS, Hsu HP, Lai MD, Yen MC, Luo YP and Chen YL: Increased expression of argininosuccinate synthetase protein predicts poor prognosis in human gastric cancer. Oncol Rep 33: 49-57, 2015.

31. Edge SB and Compton CC: The American joint committee on cancer: The 7th edition of the AJCC cancer staging manual and the future of TNM. Ann Surg Oncol 17: 1471-1474, 2010.

32. Shan YS, Fang JH, Lai MD, Yen MC, Lin PW, Hsu HP, Lin CY and Chen YL: Establishment of an orthotopic transplantable gastric cancer animal model for studying the immunological effects of new cancer therapeutic modules. Mol Carcinog 50: 739-750, 2011 
33. Shan YS, Hsu HP, Lai MD, Yen MC, Chen WC, Fang JH, Weng TY and Chen YL: Argininosuccinate synthetase 1 suppression and arginine restriction inhibit cell migration in gastric cancer cell lines. Sci Rep 5: 9783, 2015

34. Rhodes DR, Yu J, Shanker K, Deshpande N, Varambally R, Ghosh D, Barrette T, Pandey A and Chinnaiyan AM: ONCOMINE: A cancer microarray database and integrated data-mining platform. Neoplasia 6: 1-6, 2004.

35. Győrffy B, Surowiak P, Budczies J and Lànczky A: Online survival analysis software to assess the prognostic value of biomarkers using transcriptomic data in non-small-cell lung cancer. PLoS one 8: e82241, 2013.

36. Szász AM, Lánczky A, Nagy Á, Förster S, Hark K, Green JE, Boussioutas A, Busuttil R, Szabó A and Győrffy B Cross-validation of survival associated biomarkers in gastric cancer using transcriptomic data of 1,065 patients. Oncotarget 7: 49322-49333, 2016.

37. Győrffy B, Benke Z, Lánczky A, Balázs B, Szállási Z, Timár J and Schäfer R: RecurrenceOnline: An online analysis tool to determine breast cancer recurrence and hormone receptor status using microarray data. Breast Cancer Res Treat 132: 1025-1034, 2012 .

38. Takano Y, Kato Y, van Diest PJ, Masuda M, Mitomi H and Okayasu I: Cyclin D2 overexpression and lack of p27 correlate positively and cyclin E inversely with a poor prognosis in gastric cancer cases. Am J Pathol 156: 585-594, 2000.

39. Cho JY, Lim JY, Cheong JH, Park YY, Yoon SL, Kim SM, Kim SB, Kim H, Hong SW, Park YN, et al: Gene expression signature-based prognostic risk score in gastric cancer. Clin Cancer Res 17: 1850-1857, 2011.

40. D'Errico M, de Rinaldis E, Blasi MF, Viti V, Falchetti M, Calcagnile A, Sera F, Saieva C, Ottini L, Palli D, et al: Genome-wide expression profile of sporadic gastric cancers with microsatellite instability. Eur J Cancer 45: 461-469, 2009.

41. Kaptain S, Tan LK and Chen B: Her-2/neu and breast cancer. Diagn Mol Pathol 10: 139-152, 2001

42. Gravalos C and Jimeno A: HER2 in gastric cancer: A new prognostic factor and a novel therapeutic target. Ann Oncol 19: $1523-1529,2008$

43. Yu J, Leung WK, Ebert MP, Leong RW, Tse PC, Chan MW Bai AH, To KF, Malfertheiner P and Sung JJ: Absence of cyclin D2 expression is associated with promoter hypermethylation in gastric cancer. Br J Cancer 88: 1560-1565, 2003.

44. Evron E, Umbricht CB, Korz D, Raman V, Loeb DM, Niranjan B, Buluwela L, Weitzman SA, Marks J and Sukumar S: Loss of cyclin D2 expression in the majority of breast cancers is associated with promoter hypermethylation. Cancer Res 61: 2782-2787, 2001.

45. Padar A, Sathyanarayana UG, Suzuki M, Maruyama R, Hsieh JT, Frenkel EP, Minna JD and Gazdar AF: Inactivation of cyclin D2 gene in prostate cancers by aberrant promoter methylation. Clin Cancer Res 9: 4730-4734, 2003.

46. Mermelshtein A, Gerson A, Walfisch S, Delgado B, Shechter-Maor G, Delgado J, Fich A and Gheber L: Expression of D-type cyclins in colon cancer and in cell lines from colon carcinomas. Br J Cancer 93: 338-345, 2005.

47. Bartkova J, Thullberg M, Slezak P, Jaramillo E, Rubio C, Thomassen LH and Bartek J: Aberrant expression of G1-phase cell cycle regulators in flat and exophytic adenomas of the human colon. Gastroenterology 120: 1680-1688, 2001

48. Cunningham D, Allum WH, Stenning SP, Thompson JN, Van de Velde CJ, Nicolson M, Scarffe JH, Lofts FJ, Falk SJ, Iveson TJ, et al: Perioperative chemotherapy versus surgery alone for resectable gastroesophageal cancer. N Engl J Med 355: 11-20, 2006.

49. Lim L, Michael M, Mann GB and Leong T: Adjuvant therapy in gastric cancer. J Clin Oncol 23: 6220-6232, 2005.

50. Matsuura Y, Saito R, Kawagoe T, Toki N, Sugihara K and Kashimura M: Cytologic analysis of primary stomach adenocarcinoma metastatic to the uterine cervix. Acta Cytol 41: 291-294, 1997.
51. Pèrez-Montiel D, Serrano-Olvera A, Salazar LC, Cetina-Pèrez L, Candelaria M, Coronel J, Montalvo LA and de Leon DC: Adenocarcinoma metastatic to the uterine cervix: A case series. J Obstet Gynaecol Res 38: 541-549, 2012.

52. Jares P, Colomer D and Campo E: Genetic and molecular pathogenesis of mantle cell lymphoma: Perspectives for new targeted therapeutics. Nat Rev Cancer 7: 750-762, 2007.

53. Nakagawa H, Zukerberg L, Togawa K, Meltzer SJ, Nishihara T and Rustgi AK: Human cyclin D1 oncogene and esophageal squamous cell carcinoma. Cancer 76: 541-549, 1995.

54. Chen B, Zhang XY, Zhang YJ, Zhou P, Gu Y and Fan DM: Antisense to cyclin D1 reverses the transformed phenotype of human gastric cancer cells. World J Gastroenterol 5: 18-21, 1999.

55. Zhou P, Jiang W, Zhang YJ, Kahn SM, Schieren I, Santella RM and Weinstein IB: Antisense to cyclin D1 inhibits growth and reverses the transformed phenotype of human esophageal cancer cells. Oncogene 11: 571-580, 1995.

56. Seo JH, Jeong ES and Choi YK: Therapeutic effects of lentivirus-mediated shRNA targeting of cyclin D1 in human gastric cancer. BMC cancer 14: 175, 2014

57. Jiang W, Kahn SM, Zhou P, Zhang YJ, Cacace AM, Infante AS, Doi S, Santella RM and Weinstein IB: Overexpression of cyclin D1 in rat fibroblasts causes abnormalities in growth control, cell cycle progression and gene expression. Oncogene 8: 3447-3457, 1993.

58. Baldin V, Lukas J, Marcote MJ, Pagano M and Draetta G: Cyclin $\mathrm{D} 1$ is a nuclear protein required for cell cycle progression in G1. Genes Dev 7: 812-821, 1993.

59. Ma L, Wang X, Lan F, Yu Y, Ouyang X, Liu W, Xie F and Huang Q: Prognostic value of differential CCND1 expression in patients with resected gastric adenocarcinoma. Med Oncol 32: $338,2015$.

60. Stahl P, Seeschaaf C, Lebok P, Kutup A, Bockhorn M, Izbicki JR, Bokemeyer C, Simon R, Sauter G and Marx AH: Heterogeneity of amplification of HER2, EGFR, CCND1 and MYC in gastric cancer. BMC Gastroenterol 15: 7, 2015.

61. Liu SC, Bassi DE, Zhang SY, Holoran D, Conti CJ and Klein-Szanto AJ: Overexpression of cyclin D2 is associated with increased in vivo invasiveness of human squamous carcinoma cells. Mol Carcinog 34: 131-139, 2002

62. Wang W, Zhao LJ, Tan YX, Ren H and Qi ZT: MiR-138 induces cell cycle arrest by targeting cyclin D3 in hepatocellular carcinoma. Carcinogenesis 33: 1113-1120, 2012.

63. Kalish LH, Kwong RA, Cole IE, Gallagher RM, Sutherland RL and Musgrove EA: Deregulated cyclin D1 expression is associated with decreased efficacy of the selective epidermal growth factor receptor tyrosine kinase inhibitor gefitinib in head and neck squamous cell carcinoma cell lines. Clin Cancer Res 10: 7764-7774, 2004

64. Stendahl M, Kronblad A, Rydén L, Emdin S, Bengtsson NO and Landberg G: Cyclin D1 overexpression is a negative predictive factor for tamoxifen response in postmenopausal breast cancer patients. Br J Cancer 90: 1942-1948, 2004.

65. Lundgren K, Brown M, Pineda S, Cuzick J, Salter J, Zabaglo L, Howell A, Dowsett M and Landberg G: TransATAC investigators: Effects of cyclin D1 gene amplification and protein expression on time to recurrence in postmenopausal breast cancer patients treated with anastrozole or tamoxifen: A TransATAC study. Breast Cancer Res 14: R57, 2012.

66. Boku N: HER2-positive gastric cancer. Gastric Cancer 17: 1-12, 2014.

67. Deng NT, Goh LK, Wang HN, Das K, Tao J, Tan IB, Zhang SL, Lee $\mathrm{MH}, \mathrm{Wu} \mathrm{JN}, \mathrm{Lim} \mathrm{KH}$, et al: A comprehensive survey of genomic alterations in gastric cancer reveals systematic patterns of molecular exclusivity and co-occurrence among distinct therapeutic targets. Gut 61: 673-684, 2012. 